\title{
Clinical Features and Treatment Strategy of Vasculitis-Associated Diffuse Alveolar Hemorrhage
}

\author{
Ya-Chih Tien" ${ }^{1}$, Ying-Ming Chiu ${ }^{* 1,2}$, Ming-Hui Hung ${ }^{1}$, Jin-Lan Shen ${ }^{1}$, Chih-Ming Lin*3,4,5 \\ ${ }^{1}$ Division of Allergy, Immunology and Rheumatology, Department of Internal Medicine, Changhua Christian Hospital, Changhua City, Taiwan
}

${ }^{2}$ Department of Nursing, College of Nursing, HungKuang University, Taichung City, Taiwan.

${ }^{3}$ Department of Neurology, Changhua Christian Hospital, Changhua City, Taiwan.

${ }^{4}$ Department of Social Work and Child Welfare, Providence University, Taichung, Taiwan.

${ }^{5}$ Department of Medicinal Botanicals and Health Applications, Da-Yeh University, Changhua County, Taiwan

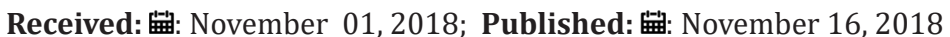

*Corresponding author: Ying-Ming Chiu, Division of Allergy, Immunology and Rheumatology, Department of Internal Medicine, Nanxiao Street, Changhua City 500-06, Taiwan

Chih- Ming Lin, Department of Neurology, Changhua Christian Hospital,Nanxiao Street, Changhua City 500-06, Taiwan

Abstract

Objectives: To summarize the clinical features and diagnostic approach of patients with vasculitis-associated diffuse alveolar hemorrhage (DAH). To investigate the optimal therapeutic strategy and highlight the effective corticosteroid dose and timing.

Methods: A retrospective chart review of the patients who were admitted to the intensive care unit (ICU) due to vasculitis-associated DAH was performed. Patient characteristics, clinical manifestations, the diagnosis of underlying etiology, treatment, and outcome were collected.

Results: During January 2015 to December 2017, seven vasculitis-associated DAH patients were reviewed. The mean age \pm SD was $53.4 \pm 18.2$ years. Five patients $(71 \%)$ were female. DAH was the initial presentation in all seven patients (100\%). All patients required immediate mechanical ventilation. As first line therapy, methylprednisolone (MTP) pulse therapy combined with cyclophosphamide pulse therapy were administered to all patients. Six patients survived. Four of them received MTP and cyclophosphamide pulse therapy shortly after admission (mean 1.6 days, range: $<1-3$ days) showed good response to therapy and were extubated successfully within ten days after ICU admission.

Conclusion: Vasculitis-associated DAH is a fatal disorder. Once the diagnosis of DAH is confirmed, intensive administration of MTP and cyclophosphamide pulse therapy initiated within 3 days of admission provide good survival and pulmonary outcome.

Keywords: Alveolar hemorrhage; Corticosteroid; Lung Diseases; Therapeutic; Vasculitis

Abbreviations: DAH: Diffuse Alveolar Hemorrhage; ICU: Intensive Care Unit; MTP: Methylprednisolone; RBCs: Red Blood Cells; ANCA: Antineutrophil Cytoplasmic Antibody; AAV: Associated Vasculitis; BAL: Broncho-Alveolar Lavage; CXR: Chest X-Ray; SD: Standard Deviation; PTU: Propylthiouracil; CRP: C-Reactive Protein; ESR: Erythrocyte Sedimentation Rate; IVIG: Intravenous Immunoglobulin

\section{Introduction}

Diffuse alveolar hemorrhage (DAH) is an acute, lifethreatening syndrome with clinical manifestations characterized by hemoptysis, dyspnea, reduced hemoglobin, and diffuse radiographic pulmonary infiltrations. The histopathology of DAH involves the accumulation of intra-alveolar red blood cells (RBCs) originating from the alveolar capillaries [1]. A broad spectrum of disorders, including immune-mediated diseases, infections, malignancies, and drugs, are all the possible underlying etiologies of DAH. The most common clinical causes of DAH include small vessel vasculitis, known as antineutrophil cytoplasmic antibody
(ANCA)-associated vasculitis (AAV), followed by Goodpasture syndrome and other collagen vascular diseases $[1,2]$.

Vasculitis-associated DAH typically presents with acute/ fulminant course and generally demonstrates high morbidity and substantial mortality [3,4]. Therefore, prompt diagnosis and aggressive treatment are required to improve survival. Recognition of vasculitis-associated DAH depends on the awareness of clinicians; once the diagnosis is established, the underlying etiology must be investigated to initiate proper management. Delayed diagnosis and insufficient treatment for the early stages of vasculitis-related 
DAH may lead to irreversible pulmonary and extra-pulmonary organ damage, particularly affecting the kidneys [5]. A combination therapy of corticosteroid, cyclophosphamide, and plasma exchange was recommended in vasculitis patients who present with severe DAH [6,7]. However, the detailed therapeutic strategy (ex. corticosteroid dosing and timing) and associated prognosis have not been reported. The timing and sufficient dose of corticosteroid for appropriate management of vasculitis-associated DAH remain a challenge for clinicians in daily practice.

The aim of this present study was to describe the clinical manifestations and prognosis of seven patients with vasculitisassociated fulminant DAH, highlighting the diagnostic approach and optimal therapeutic strategy.

\section{Materials and Methods}

We retrospectively reviewed the chart records of patients with vasculitis-associated DAH who were admitted to the intensive care unit (ICU) of Changhua Christian Hospital from January 2015 to December 2017. The diagnosis of DAH was made on the basis of at least three of the following: acute onset pulmonary symptoms (dyspnea, hemoptysis), new infiltrates on chest radiographs, reduced hemoglobin level, or bloody return on broncho-alveolar lavage (BAL) with hemosiderin-laden macrophages in the absence of Table 1: Clinical characteristics of seven patients with vasculitis-associated DAH.
.

\begin{tabular}{|c|c|c|c|c|c|c|c|}
\hline Patient & 1 & 2 & 3 & 4 & 5 & 6 & 7 \\
\hline Age/Sex & $27 / \mathrm{F}$ & $26 / \mathrm{M}$ & $56 / \mathrm{F}$ & $56 / \mathrm{F}$ & $71 / \mathrm{F}$ & $75 / \mathrm{F}$ & $63 / \mathrm{M}$ \\
\hline Diagnosis of underlying etiology & $\operatorname{IgA}-\mathrm{N}$ & GPS & $\begin{array}{l}\text { PTU } \\
-\mathrm{AAV}\end{array}$ & $\begin{array}{l}\text { PTU } \\
-\mathrm{AAV}\end{array}$ & Primary AAV & Primary AAV & Primary AAV \\
\hline \multicolumn{8}{|l|}{ Pulmonary symptoms } \\
\hline Dyspnea/duration (days) & $\mathrm{Y} /<1$ & $\mathrm{Y} / 2-3$ & $\mathrm{Y} / 1$ & $\mathrm{Y} / 1$ & $\mathrm{Y} / 1$ & $\mathrm{Y} / 1$ & $\mathrm{Y} / 1$ \\
\hline Hemoptysis/duration (days) & $\mathrm{N}$ & $\mathrm{Y} / 2-3$ & $\mathrm{Y} / 1$ & $\mathrm{~N}$ & $\mathrm{Y} / 1$ & $\mathrm{Y} / 1$ & $\mathrm{~N}$ \\
\hline Bloody sputum from endotracheal tube & Y & $\mathrm{Y}$ & $\mathrm{Y}$ & $\mathrm{Y}$ & $\mathrm{Y}$ & $\mathrm{Y}$ & $\mathrm{Y}$ \\
\hline Others & & & & $\begin{array}{l}\text { cough, } 20 \\
\text { days }\end{array}$ & & & \\
\hline Anemia on admission/Hb (g/dL) & $\mathrm{Y} / 10$ & Y/8.6 & $\mathrm{Y} / 6.6$ & $\mathrm{Y} / 6.4$ & $\mathrm{Y} / 6$ & Y/6.5 & Y/5.9 \\
\hline Reduction in $\mathrm{Hb}$ level $(\mathrm{g} / \mathrm{dL})^{\mathrm{a}}$ & 2.8 & NA & NA & NA & 1.3 & 1.4 & NA \\
\hline Abnormal chest $\mathrm{x}$-ray & $\mathrm{Y}$ & $\mathrm{Y}$ & $\mathrm{Y}$ & $\mathrm{Y}$ & $\mathrm{Y}$ & $\mathrm{Y}$ & $\mathrm{Y}$ \\
\hline Bilateral symmetric alveolar opacities & $\mathrm{N}$ & Y & $\mathrm{N}$ & $\mathrm{N}$ & $\mathrm{N}$ & $\mathrm{N}$ & $\mathrm{N}$ \\
\hline $\begin{array}{l}\text { Extensive asymmetric alveolar opacities } \\
\text { involved both upper and lower lung field }\end{array}$ & Y & $\mathrm{N}$ & Y & Y & Y & Y & Y \\
\hline Broncho-alveolar lavage & NA & Y & NA & $\mathrm{Y}$ & NA & NA & $\mathrm{Y}$ \\
\hline Suggestive of $\mathrm{DAH}^{\mathrm{b}}$ & NA & Y & NA & Y & NA & NA & Y \\
\hline Hemosiderin-laden macrophage & NA & Y & NA & $\mathrm{Y}$ & NA & NA & $\mathrm{Y}$ \\
\hline Renal involvement & $\mathrm{Y}$ & Y & $\mathrm{N}$ & $\mathrm{Y}$ & Y & Y & $\mathrm{Y}$ \\
\hline $\mathrm{Cr}$ on admission $(\mathrm{mg} / \mathrm{dL})$ & 0.42 & 10.42 & 0.53 & 2.35 & 2.13 & 1.7 & 11.54 \\
\hline Acute renal injury requiring dialysis & $\mathrm{N}$ & Y & $\mathrm{N}$ & $\mathrm{Y}$ & $\mathrm{N}$ & Y & Y \\
\hline Hematuria & $\mathrm{Y}$ & Y & Y & $\mathrm{Y}$ & $\mathrm{Y}$ & $\mathrm{Y}$ & $\mathrm{Y}$ \\
\hline Proteinuria & Y & Y & $\mathrm{N}$ & $\mathrm{Y}$ & $\mathrm{Y}$ & $\mathrm{Y}$ & Y \\
\hline Casturia & $\mathrm{N}$ & Y & $\mathrm{N}$ & $\mathrm{N}$ & $\mathrm{N}$ & $\mathrm{N}$ & $\mathrm{N}$ \\
\hline Renal biopsy & $\mathrm{Y}$ & $\mathrm{Y}$ & $\mathrm{N}$ & $\mathrm{N}$ & $\mathrm{Y}$ & $\mathrm{N}$ & $\mathrm{Y}$ \\
\hline
\end{tabular}

Note: a Reduction in hemoglobin before/after 24-48 hours after the onset of DAH, if data were available for comparison.

Cite this article: Ya-Chih Tien, Ying-Ming Chiu, Ming-Hui Hung, Jin-Lan Shen, Chih-Ming Lin. Clinical Features and Treatment Strategy of Vasculitis-Associated Diffuse Alveolar Hemorrhage. Biomed J Sci \& Tech Res 11(1)-2018.BJSTR. MS.ID.002057. DOI: $10.26717 /$ BJSTR.2018.11.002057. macroscopic airway lesions $[2,8,9]$. Data collection included patient characteristics, clinical manifestations (features of DAH, extrapulmonary organ involvement, and laboratory assessment), the diagnosis of underlying etiology, treatment, and outcome (patient survival and pulmonary outcome). The Institutional Review Board of Changhua Christian Hospital reviewed and approved this study.

\section{Statistical Analysis}

Basic demographic characteristics, clinical manifestations, laboratory test results, chest X-ray (CXR), diagnosis, treatment, and outcomes were reviewed and analyzed. Continuous variables were expressed as mean \pm standard deviation (SD) if normally distributed, or as median (range) if skewed.

\section{Results}

\section{Characteristics of Patients}

Seven DAH patients were admitted into the ICU from January 2015 to December 2017. Three patients exhibited primary AAV, two exhibited propylthiouracil (PTU)-induced AAV, one exhibited IgA-nephropathy, and the remaining one exhibited Goodpasture syndrome. The mean age \pm SD was $53.4 \pm 18.2$ years. Five patients $(71 \%)$ were female. DAH was the initial presentation for hospitalization in all seven patients (100\%). The details are shown 
${ }^{\mathrm{b}} \mathrm{DAH}$ is suggested by persistent blood on three sequential lavage aliquots from a single affected area of the lung.

AAV, ANCA-associated vasculitis; Cr, Creatinine; $\mathrm{DAH}$ diffuse alveolar hemorrhage; GPS, Goodpasture syndrome; $\mathrm{Hb}$, hemoglobin; IgA-N, IgA-nephropathy; N, No; NA, not available; PTU, propylthiouracil; Y, Yes.

\section{Clinical Manifestations}

Dyspnea was the most common symptom in all seven patients (100\%). Other pulmonary symptoms included hemoptysis (in four, 57\%) and cough (in one, 14\%). All patients required immediate mechanical ventilation, and overt bloody sputum from the endotracheal tube was observed in all patients (100\%). Renal involvement with hematuria (in seven, 100\%), proteinuria (in six, 86\%), and casturia (in one only, 14\%) was present in these patients. The mean \pm SD serum creatinine level was $4.16 \pm 4.37 \mathrm{mg}$ / dL (range: $0.42-11.54 \mathrm{mg} / \mathrm{dL}$ ). Five patients (71\%) suffered from acute renal injury and four (57\%) required hemodialysis. Renal biopsy was performed in four patients.

\section{Laboratory Data}

Three patients experienced a reduction of hemoglobin within 48 hours after onset of DAH, while the remaining four patients exhibited a low level of hemoglobin (5.9-8.6 g/dL) on admission; however, these four patients lacked previous hemoglobin data for comparison. BAL was performed in three patients (43\%); macroscopically hemorrhagic BAL fluid and microscopically hemosiderin-laden macrophages were found in all three patients. Bacterial, Mycobacterium tuberculosis, and fungal cultures, as well as virus analyses, were all negative at the onset of DAH. All patients were checked for C-reactive protein (CRP), procalcitonin, and erythrocyte sedimentation rate (ESR) on admission. The mean \pm SD values were as follows: CRP, $12.65 \pm 8.96 \mathrm{mg} / \mathrm{dL}$ (range 3.24-32.57 $\mathrm{mg} / \mathrm{dL}$ ); procalcitonin, $7.16 \pm 11.01 \mathrm{ng} / \mathrm{mL}$ (range $0.08-33.15 \mathrm{ng} /$ $\mathrm{mL}$ ); ESR, $79.71 \pm 46.67 \mathrm{~mm} / \mathrm{h}$ (range $12-140 \mathrm{~mm} / \mathrm{h}$ ).

\section{Chest Radiography}

CXRs showed bilateral alveolar opacities in all seven patients. A unique pattern involving extensive, asymmetric, dense alveolar opacities in both upper and lower parts of the lungs was present in six patients (86\%); this helped to distinguish alveolar hemorrhage from pulmonary edema, which typically presented as butterfly-like alveolar infiltration at CXR. We have included four patients with different etiologies of DAH as examples; their CXRs on admission are shown in Figure 1.

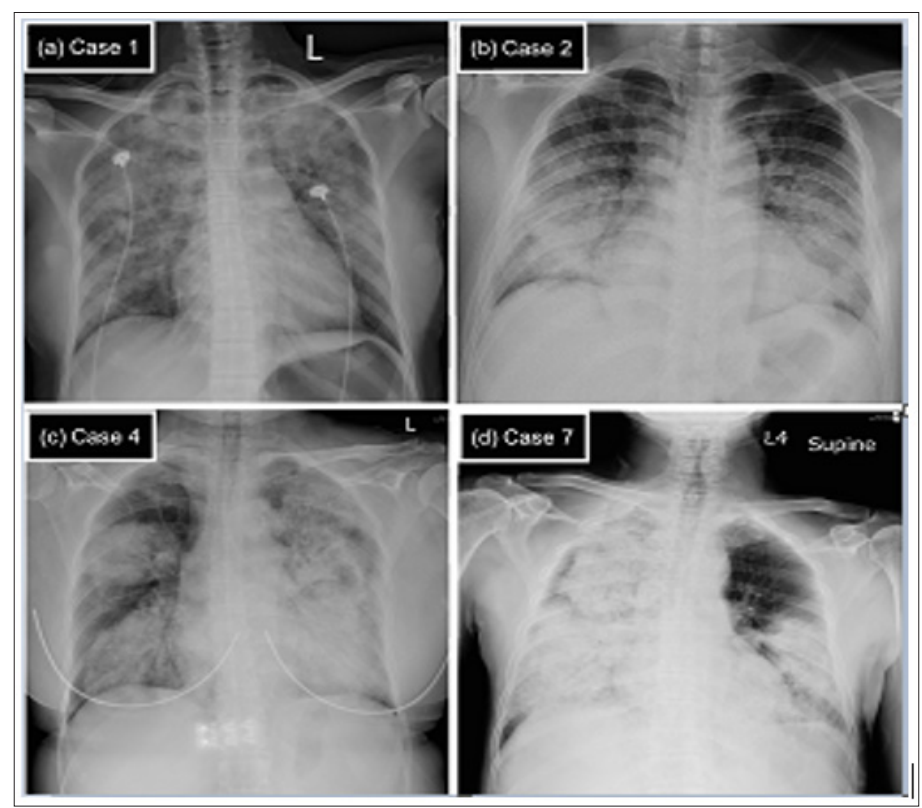

Figure 1: The chest X-ray (CXR) on admission for four diffuse alveolar hemorrhage (DAH) patients with different underlying causes.

Note: (a) Case 1. IgA-nephropathy. CXR showed extensive and asymmetric alveolar opacities in both upper and lower parts of the lungs. (b) Case 2. Goodpasture syndrome. CXR showed bilateral symmetric peri-hilar alveolar opacities that mimic pulmonary edema. (c) Case 4. Propylthiouracil-induced antineutrophil cytoplasmic antibody (ANCA)-associated vasculitis $(\mathrm{AAV})$ and $(\mathrm{d})$ Case 7. Primary AAV. For both (c) and (d), CXR revealed extensive, asymmetric, and dense alveolar opacities and consolidation that involved whole portions of the lungs.

\section{Diagnosis}

The diagnosis of underlying etiologies for DAH in seven patients was based on the clinical manifestations, laboratory test, and/or histopathology of each patient. Five patients had a diagnosis of $\mathrm{AAV}$; all were diagnosed on the basis of clinical presentation of DAH, positive serum ANCA result, and exclusion of other DAH etiologies. Two of these five patients were diagnosed with PTU-induced AAV, on the basis of drug exposure history; the other three were diagnosed with primary AAV due to the lack of associated drug exposure or other risk factors. Notably, in the remaining two patients (one with 
IgA-nephropathy and the other with Goodpasture syndrome), blood exams including anti-nuclear antibody, ANCA, and anti-glomerular basement membrane antibody were negative; they were both diagnosed by evident histopathology results on renal biopsy.

\section{Treatment and Outcome}

All patients required mechanical ventilation on admission for respiratory failure; four patients required hemodialysis for acute oliguria renal injury. Methylprednisolone (MTP) pulse therapy (750 mg per day for 3 consecutive days) combined with cyclophosphamide pulse $\left(0.5-0.75 \mathrm{mg} / \mathrm{m}^{2}\right.$, adjusted according to renal function) therapy were administered to all patients as first-line therapy. Plasma exchange was performed in six patients (86\%). Intravenous immunoglobulin (IVIG) $(0.4 \mathrm{~g} / \mathrm{kg}$ per day for 5 consecutive days) was prescribed as second-line therapy in two patients (29\%) whose illness was refractory to initial therapy. One patient with remitting-relapsing DAH received rituximab therapy (500 mg every week for 4 weeks). All patients received broad empiric antibiotics and anti-fungal therapy, although there was initially no positive culture result suggestive of major infection.

Throughout the course of hospitalization, no severe or overt infection occurred in any of the patients. One patient (case 4) died in the hospital; thus, the mortality rate was $14 \%$. Initially, this patient was treated conservatively by giving bolus corticosteroid (MTP 80 mg every eight hours) only. MTP pulse therapy was delayed until the $8^{\text {th }}$ day after ICU admission; however, the patient died on the next day after the first dose of MTP, due to uncontrolled DAH.

Six patients survived. Four of them (cases 1, 2, 3, and 5) showed good response to therapy and were extubated successfully within ten days after ICU admission; all four patients received MTP and cyclophosphamide pulse therapy shortly after admission. For those four patients, the duration from admission date to the first dose of MTP pulse was 1-3 days (mean 1.6 days, range: $<1-3$ days). The remaining two surviving patients reported prolonged ventilator dependency, although they were free from active DAH. One patient (case 7) began MTP pulse therapy 18 days after admission, followed by intensive cyclophosphamide pulse and serial sessions of plasma exchange. However, he experienced four recurrent episodes of DAH during 2 months of hospitalization. Complete remission of DAH was eventually reached after rituximab infusion. Due to prolonged endotracheal intubation, he received tracheostomy and remained ventilator-dependent. The other patient (case 6) began a combination of MTP pulse, cyclophosphamide pulse, and plasma exchange therapy on the day of admission, but her illness was refractory to those therapies; second-line therapy of IVIG was delayed until the 7th day after admission. Although DAH resolved 10 days after admission, she remained unconsciousness, likely due to prolonged hypoxia encephalopathy. Detailed treatment and prognosis information for the seven patients is summarized in Table 2.

Table 2: Treatment and outcomes of seven patients with vasculitis-associated DAH.

\begin{tabular}{|c|c|c|c|c|c|c|c|}
\hline Patient & 1 & 2 & 3 & 4 & 5 & 6 & 7 \\
\hline $\begin{array}{c}\text { Diagnosis of } \\
\text { underlying disease }\end{array}$ & IgA-N & GPS & PTU-AAV & PTU-AAV & Primary AAV & Primary AAV & Primary AAV \\
\hline Diagnostic modalities & Renal biopsy & Renal biopsy & $\begin{array}{c}\text { Drug exposure } \\
\text { Hx ANCA+ }\end{array}$ & $\begin{array}{c}\text { Drug exposure } \\
\text { Hx ANCA+ }\end{array}$ & $\begin{array}{c}\text { Renal biopsy } \\
\text { ANCA+ }\end{array}$ & ANCA+ & $\begin{array}{c}\text { Renal biopsy } \\
\text { ANCA+ }\end{array}$ \\
\hline Treatment (Tx) & MTP, CY & MTP, CY, PE & MTP, CY, PE & MTP, CY, PE & MTP, CY, PE & MTP, CY, PE, IVIG & $\begin{array}{l}\text { MTP, CY, PE, } \\
\text { IVIG, RTX }\end{array}$ \\
\hline $\begin{array}{l}\text { Duration from } \\
\text { pulmonary symptom } \\
\text { onset to } \mathrm{Tx}^{*} \text { (days) }\end{array}$ & 2 & $3 \sim 4$ & $<1$ & $\sim 20$ & 3 & $<1$ & $\sim 21$ \\
\hline $\begin{array}{l}\text { Duration from } \\
\text { admission date to Tx* } \\
\text { (days) }\end{array}$ & 2 & 1 & $<1$ & 8 & 3 & $<1$ & $\sim 18$ \\
\hline Outcome & $\begin{array}{l}\text { Extubated on } \\
\text { day 6, CR }\end{array}$ & $\begin{array}{l}\text { Extubated on } \\
\text { day 9, ESRD } \\
\text { on HD }\end{array}$ & $\begin{array}{l}\text { Extubated on } \\
\text { day } 10, C R\end{array}$ & Death & $\begin{array}{l}\text { Extubated on } \\
\text { day } 7, \text { CKD } \\
\text { stage II }\end{array}$ & $\begin{array}{l}\text { Prolonged } \\
\text { weaning due } \\
\text { to unclear } \\
\text { consciousness, } \\
\text { ESRD on HD }\end{array}$ & $\begin{array}{c}\text { Relapse DAH } \\
\text { s/p } \\
\text { tracheostomy, } \\
\text { CKD stage III }\end{array}$ \\
\hline
\end{tabular}

Note: *The first dose of methylprednisolone $750 \mathrm{mg}$ pulse therapy.

AAV, ANCA-associated vasculitis; ANCA, antineutrophil cytoplasmic antibodies; CKD, chronic kidney disease; CR, complete remission; CY, cyclophosphamide; DAH, diffuse alveolar hemorrhage; ESRD, end-stage renal disease; GPS, Goodpasture syndrome; HD, hemodialysis; Hx, history; IgA-N, IgA-nephropathy; IVIG; intravenous immunoglobulin; MTP, methylprednisolone; PE, plasma exchange; PTU, propylthiouracil; RTX, rituximab; Tx, treatment.

\section{Discussion}

This report was written to explore early and aggressive treatment strategy and association with survival and pulmonary outcome in patients with vasculitis-associated DAH. We found that corticosteroid pulse plus cyclophosphamide pulse (0.5-0.75 mg/ $\mathrm{m}^{2}$ ) in a timely manner (initiating administration within 3 days of admission) provided better survival or pulmonary outcome for patients with fulminant DAH. The corticosteroid dose should be as sufficient as MTP $750 \mathrm{mg}$ daily for at least 3 consecutive days. In contrast, delayed treatment after admission resulted in worse pulmonary outcomes, including mortality.

Although early and aggressive treatment for DAH is important for survival and prognosis, early recognition of DAH is challenging for 
clinicians. The classical triad of DAH includes hemoptysis, anemia, and asymmetric diffuse alveolar opacities at chest radiography. DAH should be suspected when at least two signs of the triad are present [10]. In our study, although all patients exhibited overt bloody sputum from the endotracheal tube, apparent hemoptysis before intubation was only present in $57 \%$ of patients. This is consistent with previous reports $[1,8,11]$. Since hemoptysis may be absent clinically, a decrease in hemoglobin without a clear cause is suggestive of DAH $[1,10]$. Further, the majority of our patients showed bilateral extensive alveolar opacities at CXRs, which were helpful for consideration of DAH. Occasionally, opacities located in peri-hilar or lower parts of both lung fields make it difficult to distinguish DAH from pulmonary edema or infection. In such situations, BAL plays an important role for the purposes of Documentation of alveolar hemorrhage, finding hemosiderin-laden macrophages from cytology analysis, and exclusion of infection [12].

Notably, all of our cases showed a varying degree of renal involvement; hematuria was the most common manifestation, followed by proteinuria. Two cases made the definite underlying etiology of DAH according to the characteristic histopathology of renal biopsy (cases 1 and 2, IgA-nephropathy and Goodpasture syndrome); both cases revealed negative findings in serum immunologic tests. This suggests that biopsy of the damaged organ helps to confirm the underlying etiology of DAH; the kidney may be an optimal organ if active sediment, such as hematuria, is present in urinalysis.

A combination of high-dose corticosteroid and cyclophosphamide or rituximab has been established as the cornerstone of induction treatment in small vessel vasculitis $[6,13]$. However, the specific timing of therapy and optimal corticosteroid dose has not been established. In our report, four patients who initiated MTP and cyclophosphamide pulse administration within 3 days of admission exhibited rapid remission from DAH within 1 week; they were extubated successfully within 2 weeks. In contrast, two patients (cases 4 and case 7) underwent delayed therapy due to missed diagnosis of vasculitis and a concern regarding possible infection. Actually, under the broad treatment of empiric antibiotics during early stages of illness, no patient experienced severe infection. We therefore report several good outcomes: first, in patients who were confirmed to have fulminant DAH without overt previous systemic diseases, vasculitis-mediated DAH remains the major cause. Second, the "right treatment dose" at the "right time" provides good prognosis for both survival and pulmonary outcome. The "right dose" means not only a relatively higher dose of corticosteroid, but the dose should be as high as the MTP pulse therapy (e.g., $750 \mathrm{mg}$ per day for at least 3 consecutive days), combined with cyclophosphamide pulse $\left(0.5-0.75 \mathrm{mg} / \mathrm{m}^{2}\right)$. The "right time" means that the administration of MTP and cyclophosphamide should be started as early as possible; we suggest that therapy within 3 days of admission would be optimal timing.

Although there has not been sufficient evidence to support the efficacy of plasmapheresis in patients with DAH, early plasmapheresis to remove pathogenic antibodies has been recognized as an effective and fast-acting adjuvant therapy in some studies [14-16].
However, there is no standard protocol of plasmapheresis for vasculitis-related DAH. In our report, six (86\%) patients received plasmapheresis, concomitant with the administration of corticosteroid. All of them showed severe organ damage at initial presentation, either acute renal injury or fulminant DAH with severe hypoxemia. We propose that prompt initiation of plasmapheresis is a good adjuvant therapy, in addition to MTP pulse therapy, for patients with fulminant vasculitis-related DAH.

There are limitations in our study. First, the heterogeneity of the underlying vasculitis of our cases may indicate a diversity of disease pathogenesis; this is compounded by variations in therapy and prognosis. Second, due to the small case number in our report, we cannot investigate other predictors or risk factors for prognosis. Third, the long-term outcome could not be explored in our study. A larger study with more patients and longer observation is required. Fourth, in Taiwan, the use of rituximab is restricted by the high cost and the policy of National Health Insurance and considered as second-line therapy for patients who were failure to cyclophosphamide treatment. Therefore, the first choice for those patients with vasculitis-related DAH was limited by the combination of MTP pulse with cyclophosphamide, rather than with rituximab in our report.

In conclusion, vasculitis-associated DAH is a fatal disorder. The most common presenting symptoms are acute dyspnea, overt bloody sputum from the endotracheal tube with reduced hemoglobin, and extensive alveolar opacities at CXR. Renal involvement is frequent and renal biopsy is helpful for evaluating the underlying etiology. Although infection should play an important role, it should not be viewed as an obstacle. Once the diagnosis of DAH is confirmed, intensive administration of MTP (e.g., $750 \mathrm{mg}$ daily for at least 3 consecutive days) and cyclophosphamide pulse therapy (e.g., 0.5$0.75 \mathrm{mg} / \mathrm{m}^{2}$, initiated within 3 days of admission) provide good survival and pulmonary outcome.

\section{References}

1. Lara AR, Schwarz MI (2010) Diffuse alveolar hemorrhage. Chest 137(5): 1164-1171.

2. Ioachimescu OC, Stoller JK (2008) Diffuse alveolar hemorrhage: diagnosing it and finding the cause. Cleve Clin J Med 75(4): 258-280.

3. Lauque D, Cadranel J, Lazor R, Pourrat J, Ronco P, et al. (2000) Microscopic polyangiitis with alveolar hemorrhage. A study of 29 cases and review of the literature. Medicine (Baltimore) 79(4): 222-233.

4. Hruskova Z, Casian AL, Konopasek P, Svobodova B, Frausova D, et al. (2013) Long-term outcome of severe alveolar haemorrhage in ANCAassociated vasculitis: a retrospective cohort study. Scand J Rheumatol 42(3): 211-214.

5. Leatherman JW, Davies SF, Hoidal JR (1984) Alveolar hemorrhage syndromes: diffuse microvascular lung hemorrhage in immune and idiopathic disorders. Medicine (Baltimore) 63(6): 343-361.

6. Yates M, Watts RA, Bajema IM, Cid MC, Crestani B, et al. (2016) EULAR/ ERA-EDTA recommendations for the management of ANCA-associated vasculitis. Ann Rheum Dis 75: 1583-1594.

7. CS Wu, TY Hsieh, KJ Yang, YJ Jan Wu, YH Chen, CH Chen, et al. (2016) Taiwan Rheumatology Association recommendations for the diagnosis and management of anti-neutrophil cytoplasmic antibody-associated vasculitis. Formosan J Rheumatol 30: 1-17.

8. West S, Arulkumaran N, Ind PW, Pusey CD (2013) Diffuse alveolar haemorrhage in ANCA-associated vasculitis. Intern Med 52(1): 5-13. 
9. Chang MY, Fang JT, Chen YC, Huang CC (2002) Diffuse alveolar hemorrhage in systemic lupus erythematosus: a single center retrospective study in Taiwan. Ren Fail 24(6): 791-802.

10. Lazor R (2011) Alveolar haemorrhage syndromes. Eur Respir Mon 54: 15-31.

11. Lin Y, Zheng W, Tian X, Zhang X, Zhang F, Dong Y (2009) Antineutrophil cytoplasmic antibody-associated vasculitis complicated with diffuse alveolar hemorrhage: a study of 12 cases. J Clin Rheumatol 15(7): 341344.

12. Olson AL, Schwarz MI (2007) Diffuse alveolar hemorrhage. Prog Respir Res Basel, Karger 36: 250-263.

13. Mukhtyar C, Guillevin L, Cid MC, Dasgupta B, De Groot K, et al. (2009) EULAR recommendations for the management of primary small and medium vessel vasculitis. Ann Rheum Dis 68(3): 310-317.

ISSN: 2574-1241

DOI: 10.26717/BJSTR.2018.11.002057

Ying-Ming Chiu, Chih- Ming Lin. Biomed J Sci \& Tech Res

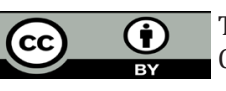

This work is licensed under Creative Commons Attribution 4.0 License

Submission Link: https://biomedres.us/submit-manuscript.php
14. Haupt ME, Pires Ervoes J, Brannen ML, Klein Gitelman MS, Prestridge AL, et al. (2013) Successful use of plasmapheresis for granulomatosis with polyangiitis presenting as diffuse alveolar hemorrhage. Pediatr Pulmonol 48(6): 614-616.

15. Aydin Z, Gursu M, Karadag S, Uzun S, Tatli E, et al. (2011) Role of plasmapheresis performed in hemodialysis units for the treatment of anti-neutrophilic cytoplasmic antibody-associated systemic vasculitides. Ther Apher Dial 15(5): 493-498.

16. Cigarran S, Castro MJ, Pousa M, Paredes S, Bernardo H, et al. (2010) Plasmapheresis in diffuse alveolar hemorrhage as perinuclear antineutrophil cytoplasmic antibody-associated vasculitis relapse on hemodialysis. Ther Apher Dial 14(3): 368-372.

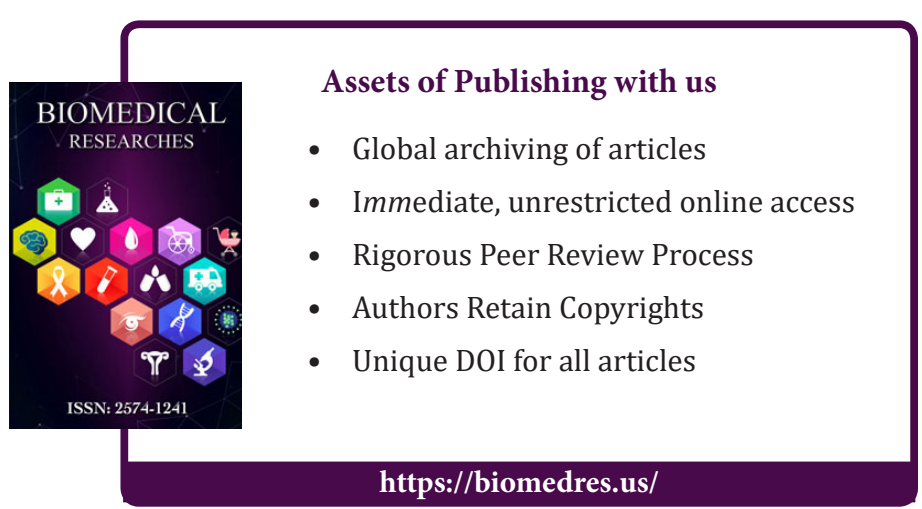

